Larsson Susanna (Orcid ID: 0000-0003-0118-0341)

Gill Dipender (Orcid ID: 0000-0001-7312-7078)

\title{
Genetically proxied growth differentiation factor 15 levels and
}

\author{
body mass index
}

Ville Karhunen PhD (ORCID: 0000-0001-6064-1588) ${ }^{1}$, Susanna C. Larsson PhD (ORCID: 0000-0003-0118-034) 2,3 $^{2}$ and Dipender Gill BMBCh PhD (ORCID: 0000-0001-7312-7078) ${ }^{1,4-}$

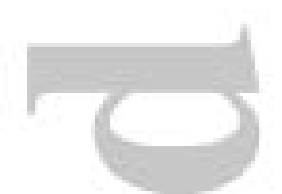

6

${ }^{1}$ Department of Epidemiology and Biostatistics, Imperial College London, London, UK.

${ }^{2}$ Unit of Cardiovascular and Nutritional Epidemiology, Institute of Environmental Medicine,

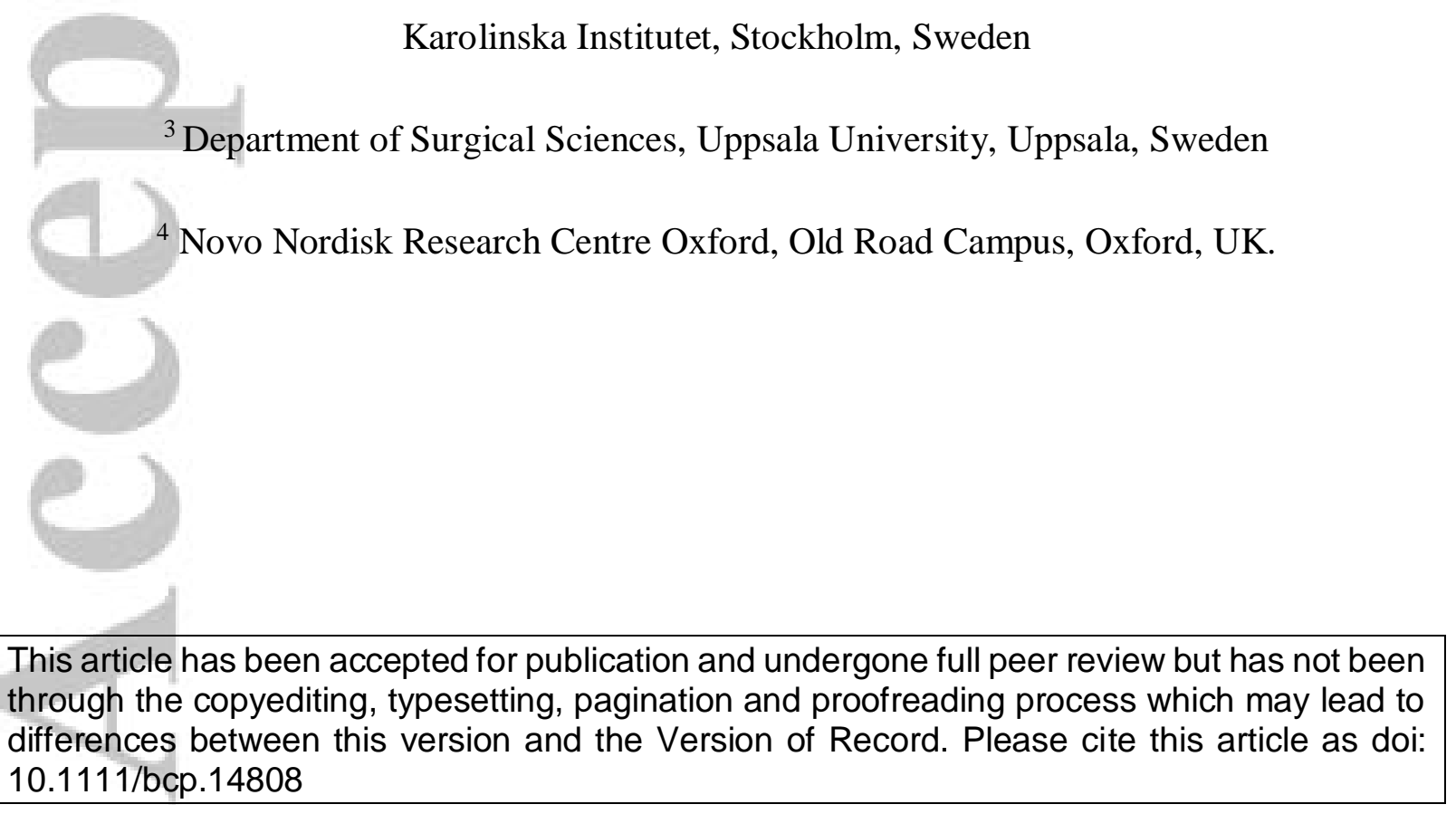


${ }^{5}$ Clinical Pharmacology and Therapeutics Section, Institute for Infection and Immunity, St George's, University of London, London, UK.

${ }^{6}$ Clinical Pharmacology Group, Pharmacy and Medicines Directorate, St George's University Hospitals NHS Foundation Trust, London, UK.

\section{Correspondence:}

Dr Ville Karhunen

Department of Epidemiology and Biostatistics, Medical School Building, St Mary's Hospital, Imperial College London, United Kingdom, W2 1PG

Telephone: +44 (0) 7375643446

Email: v.karhunen@imperial.ac.uk

Dr Dipender Gill

Department of Epidemiology and Biostatistics, Medical School Building, St Mary's Hospital, Imperial College London, United Kingdom, W2 1PG

Telephone: +44 (0) 7904843810

Email: dipender.gill@imperial.ac.uk 


\section{Keywords}

body mass index, colocalization, growth-differentiation factor 15, Mendelian randomization, type 2 diabetes

\section{Principal Investigator Statement}

This study did not perform any intervention on human subjects and, as such, no Principal Investigator Statement is required.

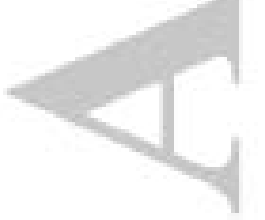

\section{Data availability}

All data used in this study are publicly available, with the relevant citations detailed.

\section{Funding}

DG and VK are supported by the British Heart Foundation Research Centre of Excellence (RE/18/4/34215) at Imperial College London. DG is supported by a National Institute for Health Research Clinical Lectureship (CL-2020-16-001) at St. George's, University of London. SCL acknowledges research support from the Swedish Research Council (Vetenskapsrådet, 2019-00977), Swedish Heart-Lung Foundation (Hjärt-Lungfonden, 20190247), and Swedish Research Council for Health, Working Life and Welfare (Forte, 2018-00123).

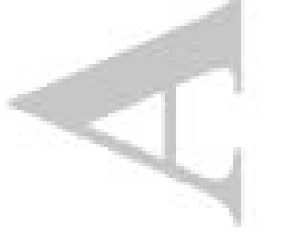




\section{Conflict of Interest Statement}

DG is employed part-time by Novo Nordisk and has received consultancy fees from Abbott

Laboratories. The remaining authors declare that there are no relationships or activities that might bias, or perceived to bias, this work.

\section{Author Contributions}

All authors substantially contributed to the conception and design of the work. VK conducted the statistical analyses and drafted the manuscript. All authors interpreted the data, critically revised the manuscript and provided final approval of the version to be submitted. VK and DG are the guarantors of the work.

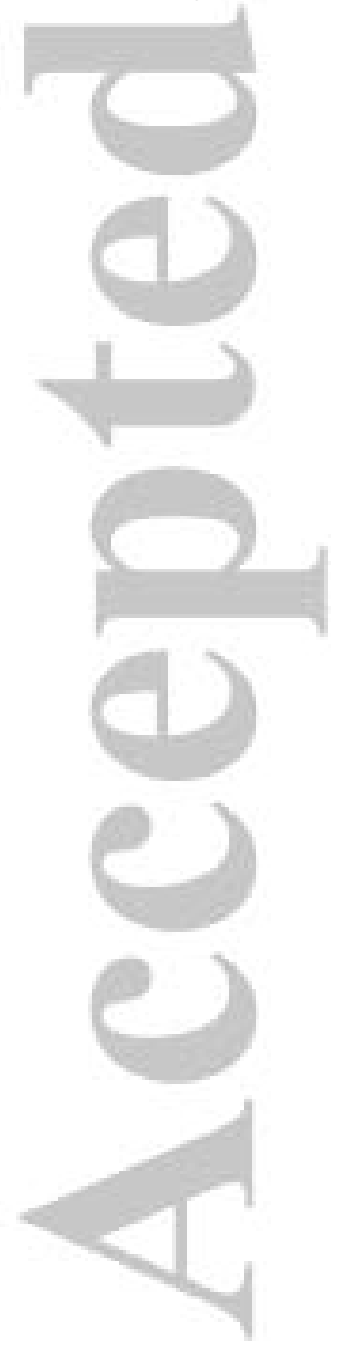




\section{What is already known about this subject}

- Growth-differentiation factor 15 is an inflammatory cytokine involved in energy homeostasis that is being pursued as a drug target for obesity.

- Circulating levels of growth-differentiation factor 15 are acutely increased by the type 2 diabetes medication metformin, resulting in reduced appetite and weight loss.

- However, the effect of chronically elevated circulating growth-differentiation factor 15 levels on body weight is not known.

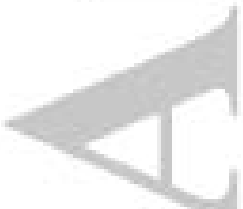

\section{What this study adds}

- Using human data, this study provides genetic evidence supporting that chronically elevated circulating growth-differentiation factor 15 levels increase body mass index.

- There was no genetic evidence to support bi-directional effects, or that chronically elevated growth-differentiation factor 15 levels directly affect liability to type 2 diabetes.

- These results contrast the body mass index lowering effects of an acute increase in growth-differentiation factor 15 levels observed after metformin use.

- These findings have direct implications for informing pharmacological strategies aimed at targeting growth-differentiation factor 15 levels for weight loss. 


\begin{abstract}
Growth-differentiation factor 15 (GDF15) is an inflammatory cytokine involved in energy homeostasis that is being pursued as a drug target for obesity. Its circulating levels are acutely increased by the type 2 diabetes medication metformin, resulting in reduced appetite and weight loss. We identified a genetic variant at the GDF15 gene to proxy a small, lifelong increase in circulating GDF15 levels, and leveraged it in colocalization and Mendelian randomization analyses to investigate the effects of chronically elevated GDF15 levels on body mass index (BMI) and type 2 diabetes liability. The results provide human genetic evidence supporting that chronically elevated GDF15 levels increase BMI. There was no genetic evidence to support bi-directional effects, or that chronically elevated GDF15 levels directly affect liability to type 2 diabetes. Our results contrast the BMI lowering effects of an acute increase in GDF15 levels observed after metformin use. These findings have direct implications for informing pharmacological strategies aimed at targeting GDF15 levels for
\end{abstract} weight loss. 


\section{Introduction}

Growth-differentiation factor 15 (GDF15) is an inflammatory cytokine involved in energy homeostasis that is being pursued as a drug target for obesity ${ }^{1}$. Its circulating levels are acutely increased by the type 2 diabetes medication metformin, resulting in reduced appetite and weight $\operatorname{loss}^{2}$. However, the effect of chronically elevated circulating GDF15 levels on body weight is not known.

Here, we identified a genetic variant at the GDF15 gene to proxy a small, lifelong increase in circulating GDF15 levels, and leveraged it in genetic analyses to investigate the effects of chronically elevated GDF15 levels on body mass index (BMI) and type 2 diabetes liability.

\section{Cothods}

Colocalization and Mendelian randomization analyses were performed. Briefly, colocalization compares the genetic associations for two traits within a given genetic locus, investigating whether the data supports a model with a shared causal variant for both traits ${ }^{3}$. In Mendelian randomization, genetic variants that proxy the effect of varying the exposure are used to investigate its effect on an outcome ${ }^{4}$. Our choice of genetic proxy for the effect of GDF15 signalling was restricted to a variant at the GDF15 locus, to reduce the risk of bias related to pleiotropic associations unrelated to $\mathrm{GDF} 15^{5}$. Further details are provided in the Supplementary Methods.

Summary statistics data for the genetic associations of single-nucleotide polymorphisms with each trait were obtained from publicly available large-scale genomewide association studies (GWAS) performed on individuals of European ancestries. These 
data can be obtained from the original studies, as detailed below. Genetic associations with circulating GDF15 levels were based on 3,301 healthy individuals ${ }^{6}$, genetic associations with BMI were obtained from a GWAS meta-analysis of 806,834 individuals ${ }^{7}$, and genetic associations with type 2 diabetes liability were based on a GWAS meta-analysis of 74,124 cases and 824,006 controls ${ }^{8}$. Relevant ethical approval and participant consent were obtained by the original studies.

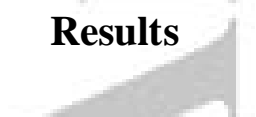

Colocalization analysis within the GDF15 gene supported the model with a shared causal genetic variant (posterior probability > 0.99; Figure) for GDF15 levels and BMI, with rs16982345 identified as the most likely shared causal variant $\left(\mathrm{p}=5.8 \times 10^{-99}\right.$ for the association with GDF15 levels, and $\mathrm{p}=4.4 \times 10^{-9}$ for the association with BMI). In Mendelian randomization analysis using the rs16982345 variant, genetically proxied higher circulating GDF15 levels were associated with increased BMI (change in standard deviation [SD] units per one SD increase in GDF15 levels: 0.021 [95\% confidence interval 0.014 to 0.028], $\left.\mathrm{p}=4.4 \times 10^{-9}\right)$. Exploring bi-directional effects, there was no evidence of genetically proxied BMI being associated with GDF15 levels (change in SD units per one SD increase in BMI [95\% confidence interval]: inverse-variance weighted estimate 0.005 [-0.133 to 0.143$]$, $p=0.95$; weighted median estimate: $-0.059[-0.260$ to 0.143$], p=0.57$; MR-Egger estimate 0.104 [-0.485 to 0.277$], \mathrm{p}=0.59$; MR-Egger intercept test for directional pleiotropy: $\mathrm{p}=$ 0.55). There was no strong evidence for colocalization of GDF15 levels and type 2 diabetes liability (posterior probability $=0.16$ ) 


\section{Discussion}

Using human data, we provide genetic evidence supporting the notion that chronically elevated GDF15 levels increase BMI. There was no genetic evidence to support bi-directional effects, or that chronically elevated GDF15 levels directly affect liability to type 2 diabetes.

Our results contrast the BMI lowering effects of an acute increase in GDF15 levels observed after metformin use ${ }^{2}$. There are a number of possible explanations for this discrepancy, including that metformin may be affecting BMI though mechanisms unrelated to GDF15. Long-term elevation of circulating GDF15 levels may also contrast the effects of acute increases in GDF15 and lead to desensitization of the GDF15 receptor and reduced signalling ${ }^{9}$. Alternative possibilities include compensatory changes in other hormones and behaviour (including dietary intake) as result of elevated circulating GDF15 levels. Furthermore, it is possible that GDF15 affects foetal and childhood development, thus impacting adult BMI through this mechanism.

The use of both colocalization and Mendelian randomization in this study provide complementary evidence supporting causal effects of chronically elevated GDF15 levels on BMI. As genetic variants are randomly allocated at conception, the Mendelian randomization paradigm is less susceptible to the confounding and reverse causation that that can hinder causal inference in observational studies. A previous Mendelian randomization analysis exploring the effect of circulating GDF15 levels on BMI used variants from throughout the genome in their main analysis ${ }^{10}$. While the greater number of variants generated through this strategy allows for the incorporation of Mendelian randomization statistical sensitivity analyses, this approach also risks including variants that have pleiotropic associations unrelated to GDF15, which can consequently bias the Mendelian randomization analysis ${ }^{5}$. Previous Mendelian randomization studies exploring the effect of circulating GDF15 have 
also used GDF15 genetic association estimates that were adjusted for systolic blood pressure, antihypertensive medication use, diabetes mellitus and smoking status ${ }^{1011}$, which can introduce collider bias ${ }^{12}$. Another previous study used a genetic variant related to GDF15 gene expression in whole blood to proxy the effect of varying GDF15 circulating levels ${ }^{13}$. However, colocalization analysis was not performed in this work, so it is unclear if genetic confounding was introducing bias into consequent Mendelian randomization analysis ${ }^{13}$. Such issues potentially explain the discrepancy in the results of these previous studies with our current work, which did not suffer from the same limitations.

As a limitation of our current work, the genetic associations were derived from individuals of European ancestries, and therefore our results may not generalize to other ethnic groups. A further limitation is that we used BMI as a measure of obesity. However, BMI may also be elevated in the context of increased lean mass and body water content, and is not always reflective of increased body fat.

In conclusion, this genetic analysis found robust evidence to support that, in contrast to acute elevations in GDF15 levels, chronically elevated GDF15 levels increase BMI. These findings may be used to inform the design of pharmacological strategies aimed at targeting GDF15 for weight loss. 


\section{References}

1. Tsai VWW, Husaini Y, Sainsbury A, et al. The MIC-1/GDF15-GFRAL Pathway in Energy Homeostasis: Implications for Obesity, Cachexia, and Other Associated Diseases. Cell Metabolism 2018;28(3):353-68. doi: https://doi.org/10.1016/j.cmet.2018.07.018

2. Coll AP, Chen M, Taskar P, et al. GDF15 mediates the effects of metformin on body weight and energy balance. Nature 2020;578(7795):444-48. doi: 10.1038/s41586019-1911-y

3. Giambartolomei C, Vukcevic D, Schadt EE, et al. Bayesian Test for Colocalisation between Pairs of Genetic Association Studies Using Summary Statistics. PLOS Genetics 2014;10(5):e1004383. doi: 10.1371/journal.pgen.1004383

4. Davey Smith G, Ebrahim S. 'Mendelian randomization': can genetic epidemiology contribute to understanding environmental determinants of disease?*. International Journal of Epidemiology 2003;32(1):1-22. doi: 10.1093/ije/dyg070

5. Gill D, Georgakis MK, Walker VM, et al. Mendelian randomization for studying the effects of perturbing drug targets [version 2; peer review: awaiting peer review]. Wellcome Open Res 2021;6(16):(https://doi.org/10.12688/wellcomeopenres.6544.2).

6. Sun BB, Maranville JC, Peters JE, et al. Genomic atlas of the human plasma proteome. Nature 2018;558(7708):73-79. doi: 10.1038/s41586-018-0175-2

7. Pulit SL, Stoneman C, Morris AP, et al. Meta-analysis of genome-wide association studies for body fat distribution in 694649 individuals of European ancestry. Human Molecular Genetics 2018;28(1):166-74. doi: 10.1093/hmg/ddy327

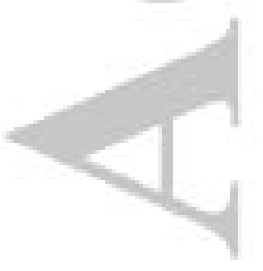


8. Mahajan A, Taliun D, Thurner M, et al. Fine-mapping type 2 diabetes loci to single-variant resolution using high-density imputation and islet-specific epigenome maps. Nature Genetics 2018;50(11):1505-13. doi: 10.1038/s41588-018-0241-6

9. Eddy AC, Trask AJ. Growth Differentiation Factor-15 and Its Role in Diabetes and Cardiovascular Disease. Cytokine \& Growth Factor Reviews 2020 doi: https://doi.org/10.1016/j.cytogfr.2020.11.002

10. Au Yeung SL, Luo S, Schooling CM. The impact of GDF-15, a biomarker for metformin, on the risk of coronary artery disease, breast and colorectal cancer, and type 2 diabetes and metabolic traits: a Mendelian randomisation study. Diabetologia 2019;62(9):1638-46. doi: 10.1007/s00125-019-4913-2

11. Cheung CL, Tan KCB, Au PCM, et al. Evaluation of GDF15 as a therapeutic target of cardiometabolic diseases in human: A Mendelian randomization study. EBioMedicine 2019;41:85-90. doi: 10.1016/j.ebiom.2019.02.021

12. Holmes MV, Davey Smith G. Problems in interpreting and using GWAS of conditional phenotypes illustrated by 'alcohol GWAS'. Mol Psychiatry 2018 doi: 10.1038/s41380018-0037-1

13. Moon JS, Goeminne LJE, Kim JT, et al. Growth differentiation factor 15 protects against the aging-mediated systemic inflammatory response in humans and mice. Aging Cell 2020;19(8):e13195. doi: 10.1111/acel.13195 

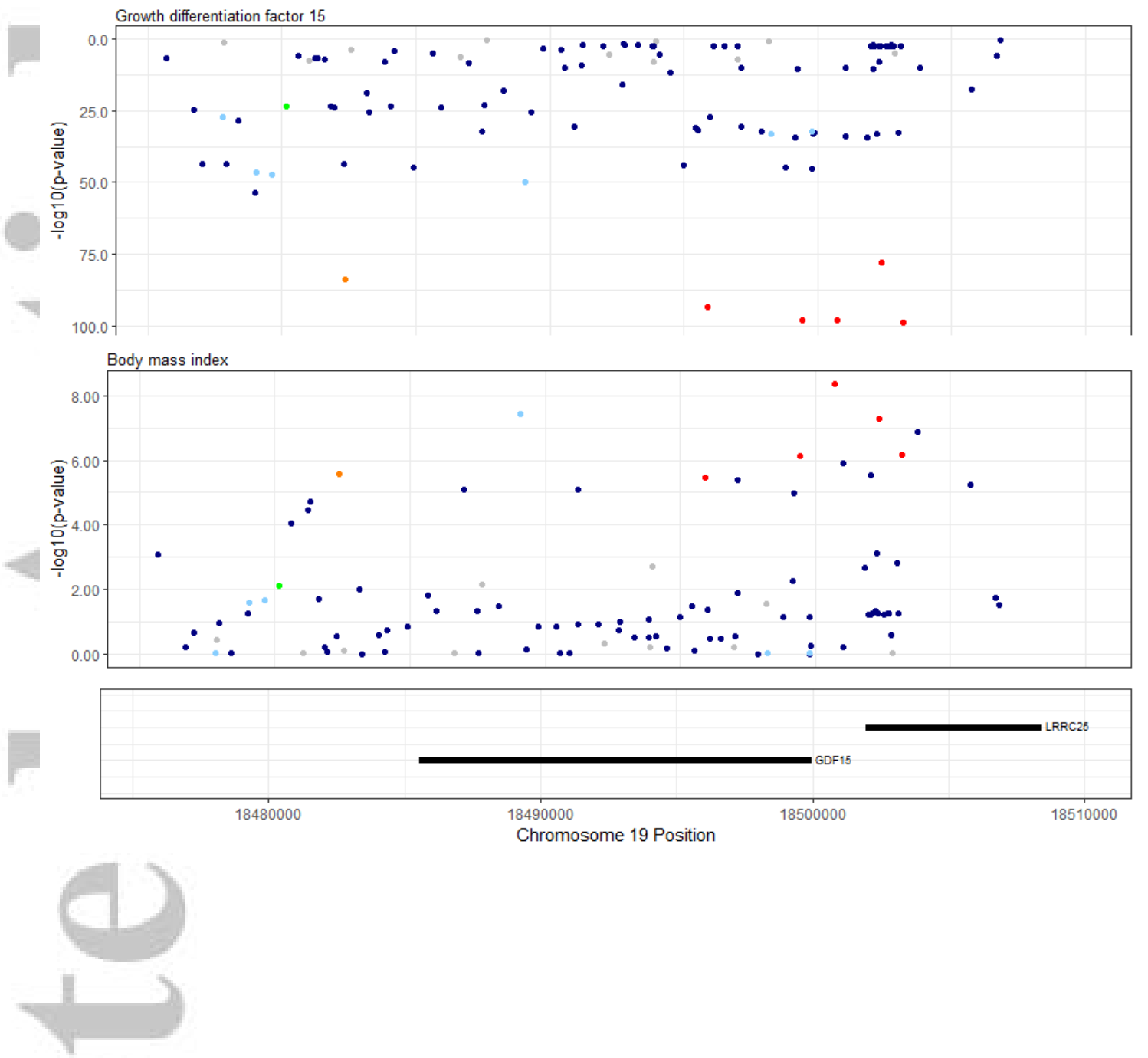

Figure. Colocalization plot of genetic associations for circulating growth differentiation factor 15 levels and body mass index within $\pm 10 \mathrm{~kb}$ of GDF15 gene. LD = linkage disequilibrium $r^{2}$ with rs16982345, the variant identified as the most likely shared causal variant. 